\title{
The prognostic value of the nodal ratio in N1 breast cancer
}

\author{
Tae Jin Han', Eun Young Kang ${ }^{1}$, Wan Jeon', Sung-Won Kim², Jee Hyun Kim², Yu Jung Kim², So Yeon Park², \\ Jae Sung $\mathrm{Kim}^{1}$ and In Ah Kim ${ }^{1,2^{*}}$
}

\begin{abstract}
Background: Although the nodal ratio (NR) has been recognized as a prognostic factor in breast cancer, its clinical implication in patients with 1-3 positive nodes (N1) remains unclear. Here, we evaluated the prognostic value of the NR and identified other clinico-pathologic variables associated with poor prognosis in these patients.

Methods: We analyzed 130 patients with N1 invasive breast cancer who were treated at Seoul National University Bundang Hospital from March 2003 to December 2007. Disease-free survival (DFS), locoregional recurrence-free survival (LRRFS), and distant metastasis-free survival (DMFS) were compared according to the NR with a cut-off value of 0.15 .

Results: We followed patients' recovery for a median duration of 59 months. An NR $>0.15$ was found in $23.1 \%$ of patients, and a median of 18 nodes were dissected per patient (range 1-59). The NR was statistically independent from other prognostic variables, such as patient age, $T$ stage, extent of surgery, pathologic factors in the chi square test. On univariate analysis, patients with a NR $>0.15$ had significantly lower 5-year LRRFS (88.7\% vs. 97.9\%, $p=$ $0.033)$ and 5 -year DMFS (81.3\% vs. $96.4 \%, p=0.029)$ and marginally lower 5 -year DFS (81.3\% vs. 94.0\%, $p=0.069)$ than those with a NR $\leq 0.15$, respectively. Since the predictive power of the NR was found to differ with diverse clinical and pathologic variables, we performed adjusted analysis stratified by age, pathologic characteristics, and adjuvant treatments. Only young patients with a NR $>0.15$ showed significantly lower DFS $(p=0.027)$ as well as those presenting an unfavorable pathologic profile such as advanced T stage $(p=0.034)$, histologic grade $3(p=$ 0.034), positive lymphovascular invasion ( $p=0.037)$, involved resection margin $(p=0.007)$, and no chemotherapy $(p=0.014)$ or regional radiotherapy treatment $(p=0.039)$. On multivariate analysis, a $N R>0.15$ was significantly associated with lower DFS $(p=0.043)$ and DMFS $(p=0.012)$, but not LRRFS $(p=0.064)$.
\end{abstract}

Conclusions: A NR $>0.15$ was associated with an increased risk of recurrence, especially in young patients with unfavorable pathologic profiles.

Keywords: breast cancer, N1, nodal ratio, prognostic factor

\section{Background}

The presence of axillary lymph node metastasis is one of the most important factors affecting prognosis in patients with breast cancer [1]. According to the current 7th edition of the American Joint Committee on Cancer staging system, $\mathrm{N}$ stage in breast cancer is solely determined by the number of positive nodes [2]. In patients with inappropriately dissected axillary nodes, however, a discrepancy

\footnotetext{
* Correspondence: inah228@snu.ac.kr

'Department of Radiation Oncology, Seoul National University, Bundang

Hospital, 166 Gumiro Seongnamsi Kyeonggido, 463-707, Korea

Full list of author information is available at the end of the article
}

may exist between the absolute number of positive nodes and the substantive extent of axillary node metastasis [3]. Therefore, the nodal ratio (NR), defined as the absolute number of involved nodes/number of excised nodes, has been suggested to address this discrepancy [4]. Recent studies have shown the prognostic value of the NR and even proposed the possibility of NR as an alternative or a complement to $\mathrm{N}$ staging in node-positive breast cancer [5-13]. However, no consensus has been reached for the appropriate criteria to discriminate between low- and high-risk groups of NR for breast cancer with 1-3 positive nodes.

\section{() Biomed Central}

() 2011 Han et al; licensee BioMed Central Ltd. This is an Open Access article distributed under the terms of the Creative Commons Attribution License (http://creativecommons.org/licenses/by/2.0), which permits unrestricted use, distribution, and reproduction in any medium, provided the original work is properly cited. 
In the current study, we evaluated the prognostic value of the NR and identified other clinico-pathologic variables associated with poor prognosis in N1 breast cancer patients.

\section{Methods}

\section{Patients}

We retrospectively analyzed 130 patients with N1 invasive breast cancer who were treated at Seoul National University Bundang Hospital (SNUBH) from March 2003 to December 2007. Patients who had received neoadjuvant chemotherapy prior to surgery were excluded. We collected not only treatment modality information such as type of surgery, type of systemic treatment, and radiation field, but also detailed clinicopathologic prognostic factors such as age, pathologic stage, histologic type and grade, number of excised and positive nodes, estrogen/progesterone receptor (ER/PR) status, human epithelial growth factor receptor family 2 (HER2) status, presence of extracapsular extension (ECE), presence of lymphovascular invasion (LVI), and resection margin status. A close margin was defined as the presence of invasive carcinoma within $2 \mathrm{~mm}$ of the surgical margin of resection.

\section{Patient grouping according to the nodal ratio}

We categorized the patients into two NR groups: low NR (LNR; $\leq 0.15)$ and high NR (HNR; > 0.15). Disease-free survival (DFS), locoregional recurrence-free survival (LRRFS), and distant metastasis-free survival (DMFS) were compared between groups. We defined locoregional recurrence as the first site of recurrence involving residual breast or chest wall (local) tissue and/or axillary, supra- or infraclavicular, and internal mammary nodes (regional). For cases in which locoregional recurrence and distant metastasis simultaneously occurred, we counted both failure patterns.

\section{Statistical analysis}

To make comparisons between the two groups, we used the chi-square test or Fisher's exact test for categorical data and independent sample t-test for continuous data. The Kaplan-Meier method was used for DFS, LRRFS, and DMFS probability, and survival according to different variables was compared by the log-rank test. The Cox proportional hazard method was used to perform multivariate analysis for predictors of survival. We included variables that showed significance in the univariate analysis or were otherwise were considered to be confounders in the multivariate analysis. All statistical analyses were performed with Statistical Package for the Social Sciences (version 17.0; SPSS, Chicago, IL). We considered $p$ values equal to or less than 0.05 to be statistically significant.

\section{Results}

\section{Patient and tumor characteristics}

Of the 130 patients, the LNR group included 100 patients and the HNR group included 30 patients. Patient characteristics for these two groups are summarized in Table 1. The median number of excised nodes per patient was 18 (range, 1-59) for both groups combined, and was significantly higher in the LNR group than in the HNR group (20 vs. 7, p < 0.001). RT was used to treat 46 (46\%) LNR patients and 20 (66.7\%) HNR patients; among these, regional RT was more frequently used in the HNR group (50.0\% vs. $10.9 \%, \mathrm{p}=0.001)$. The local RT field consisted of the whole breast or chest wall only. In contrast, supraclavicular lymph nodes and/or internal mammary lymph nodes were included in the locoregional RT field. Chemotherapy was used to treat 92 (92\%) LNR patients and $25(83.3 \%)$ HNR patients. Taxane-containing regimens such as AC (adriamycin and cyclophosphamide) were most frequently prescribed. The tumor characteristics in the two groups are summarized in Table 2. Infiltrating ductal carcinoma was the most frequent tumor histology in both groups, but was more dominant in the LNR group $(92.0 \%$ vs. $73.3 \%, \mathrm{p}=0.011)$. The NR was a statistically independent variable from other prognostic variables including patient age, extent of surgery, and pathologic factors such as ECE, LVI, tumor grade, margin status, ER/PR, and HER2 status.

\section{Follow-up and patterns of failure}

We followed patients' recovery for a median duration of 59 months (range, 10-89 months) for both groups although the LNR group had a longer duration of follow-up ( $\mathrm{p}=0.013$ ). Both groups showed distant metastasis as the dominant failure pattern in eight of nine patients who experienced any failures. These details are summarized in Table 3.

\section{Univariate analysis of different prognostic factors}

The univariate analysis results for prognostic factors are summarized in Table 4. According to the univariate analysis, patients with a NR $>0.15$ had significantly lower 5-year LRRFS (88.7\% vs. 97.9\%, p = 0.033) and 5year DMFS (81.3\% vs. $96.4 \%, \mathrm{p}=0.029)$ and marginally lower 5-year DFS (81.3\% vs. $94.0 \%, \mathrm{p}=0.069)$ than those with a NR $\leq 0.15$ (Figure 1).

\section{The effect of NR on DFS stratified by other prognostic factors}

Since the prognostic power of the NR was found to differ according to diverse clinical and pathologic variables, we performed adjusted analysis stratified by age, pathologic characteristics, and adjuvant treatments. The HNR group showed significantly lower 5-year DFS exclusively 


\begin{tabular}{|c|c|c|c|c|}
\hline & No. of patients & & & \\
\hline & $\begin{array}{l}N R<0.15 \\
(n=100)\end{array}$ & $\begin{array}{c}\mathrm{NR}>0.15 \\
(\mathrm{n}=30)\end{array}$ & $p$ value & $\begin{array}{c}\text { Total } \\
(n=130)\end{array}$ \\
\hline Age (years) & & & 0.225 & \\
\hline median (range) & $46(25-79)$ & $50(32-82)$ & & $47(25-82)$ \\
\hline Excised LN (No.) & & & $<0.001$ & \\
\hline median (range) & $20(7-59)$ & $7(1-18)$ & & $18(1-59)$ \\
\hline Breast resection & & & 0.290 & \\
\hline $\mathrm{BCS}$ & $49(49.0)$ & $18(60.0)$ & & $67(51.5)$ \\
\hline MRM & $51(51.0)$ & $12(40.0)$ & & $63(48.5)$ \\
\hline LN resection & & & 0.071 & \\
\hline SLNB only & $3(3.0)$ & $4(13.3)$ & & $7(5.4)$ \\
\hline$S L N B+A L N D$ & $68(68.0)$ & $20(66.7)$ & & $88(67.7)$ \\
\hline ALND & $29(29.0)$ & $6(20.0)$ & & $35(26.9)$ \\
\hline Radiotherapy & & & 0.047 & \\
\hline no & $54(54.0)$ & $10(33.3)$ & & $64(49.2)$ \\
\hline yes & $46(46.0)$ & $20(66.7)$ & & $66(50.8)$ \\
\hline Extent of radiotherapy & & & 0.001 & \\
\hline local & $41(89.1)$ & $10(50.0)$ & & $51(77.2)$ \\
\hline locoregional & $5(10.9)$ & $10(50.0)$ & & $15(22.8)$ \\
\hline Chemotherapy & & & 0.176 & \\
\hline no & $8(8.0)$ & $5(16.7)$ & & $13(10.0)$ \\
\hline yes & $92(92.0)$ & $25(83.3)$ & & $117(90.0)$ \\
\hline Regimen & & & 0.141 & \\
\hline CMF & $12(13.0)$ & $7(28.0)$ & & $19(16.2)$ \\
\hline $\mathrm{FEC} / \mathrm{FAC}$ & $31(33.7)$ & $9(36.0)$ & & $40(34.2)$ \\
\hline $\mathrm{ACT}$ & $49(53.3)$ & $9(36.0)$ & & $58(49.6)$ \\
\hline
\end{tabular}

Abbreviations: LN, lymph node; BCS, breast-conserving surgery; MRM, modified radical mastectomy; SLNBx, sentinel lymph node biopsy; ALND, axillary lymph node dissection; CMF, cyclophosphamide/methotrexate/5-fluorouracil; FEC, 5-FU/epirubicin/cyclophosphamide; FAC, 5-FU/adriamycin/cyclophosphamide; ACT, adriamycin/cyclophosphamide/paclitaxel

in those presenting an unfavorable clinico-pathologic profile: young age $(\mathrm{p}=0.027)$, advanced $\mathrm{T}$ stage $(\mathrm{p}=$ $0.034)$, high grade $(\mathrm{p}=0.034)$, the presence of LVI $(\mathrm{p}=$ $0.037)$, involved resection margin $(p=0.007)$ and the lack of chemotherapy $(\mathrm{p}=0.014)$ or regional $\mathrm{RT}(\mathrm{p}=$ 0.039; Table 5). The DFS curves according to NR with and without regional RT are presented in Figure 2.

\section{Multivariate analysis of different prognostic factors}

According to the multivariate analysis, a NR $>0.15$ was significantly associated with lower DFS $(\mathrm{p}=0.043)$ and DMFS $(p=0.012)$ but not LRRFS ( $p=0.064$; Table 6$)$. Patients not treated with chemotherapy showed a tendency of increased distant metastasis.

\section{Discussion}

The prognostic value of NR is supported by several studies [4-13]. Vinh-Hung et al. reported the superiority of NR over pN stage in predicting disease-specific survival, and Danko et al. revealed that the prognostic value of NR for disease-free survival remained significant even when stratified by pN stage $[8,10]$. Recently, Ahn et al. showed that NR is a better predictor of disease-free survival than $\mathrm{pN}$ stage, especially in patients with high-risk features such as young age, HER2-enriched or triplenegative tumor, and recommended that NR should be preferentially considered in decision making for adjuvant treatment [13].

Although most studies used a value between 0.20 and 0.25 as a minimal cut-off threshold to distinguish risk groups, there is no consensus on which value is the most reliable [5-14]. We used 0.15 as a cut-off value, which may be considered somewhat low. Because the number of positive nodes is inevitably limited in the N1 category, however, the distribution of the NR is strongly affected by the number of nodes sampled. While other studies have focused on patients with between 10 and 16 excised nodes, the present study investigated patients with a median of 18 excised nodes.

Extensive data suggest that other clinico-pathologic findings also can predict an increased risk of locoregional recurrence and even distant metastasis, such as young age, higher histologic grade, negative hormone receptors, presence of ECE, presence of LVI, and 
Table 2 Tumor characteristics

\begin{tabular}{|c|c|c|c|c|}
\hline & No. of patients & & & \\
\hline & $\begin{array}{l}N R \leq 0.15 \\
(n=100)\end{array}$ & $\begin{array}{c}\mathrm{NR}>0.15 \\
(\mathrm{n}=30)\end{array}$ & $p$ value & $\begin{array}{c}\text { Total } \\
(n=130)\end{array}$ \\
\hline Histology & & & 0.011 & \\
\hline IDC & $92(92.0)$ & $22(73.3)$ & & $114(87.7)$ \\
\hline others & $8(8.0)$ & $8(26.7)$ & & $16(12.3)$ \\
\hline T stage & & & 0.166 & \\
\hline $\mathrm{T} 1 \mathrm{a} / \mathrm{T} 1 \mathrm{~b}$ & $8(8.0)$ & $6(20.0)$ & & $14(10.8)$ \\
\hline T1c & $42(42.0)$ & $12(40.0)$ & & $54(41.5)$ \\
\hline $\mathrm{T} 2$ & $49(49.0)$ & $10(33.3)$ & & $59(45.4)$ \\
\hline T3 & $1(1.0)$ & $2(6.7)$ & & $3(2.3)$ \\
\hline ER & & & 0.459 & \\
\hline$(-)$ & $23(23.0)$ & $5(16.7)$ & & $28(21.5)$ \\
\hline$(+)$ & 77 (77.0) & $25(83.3)$ & & $102(78.5)$ \\
\hline PR & & & 0.155 & \\
\hline$(-)$ & $41(41.0)$ & $8(26.7)$ & & $49(37.7)$ \\
\hline$(+)$ & $59(59.0)$ & $22(73.3)$ & & $81(62.3)$ \\
\hline HER2 & & & 0.866 & \\
\hline$(-)$ & $72(72.0)$ & $25(83.3)$ & & $107(82.3)$ \\
\hline$(+)$ & $18(18.0)$ & $5(16.7)$ & & $23(17.7)$ \\
\hline unknown & $10(10.0)$ & $0(0.0)$ & & \\
\hline ECE & & & 0.183 & \\
\hline$(-)$ & $74(74.0)$ & $22(73.4)$ & & $97(74.6)$ \\
\hline$(+)$ & $26(26.0)$ & $7(23.3)$ & & $33(25.4)$ \\
\hline unknown & $0(0.0)$ & $1(3.3)$ & & \\
\hline LVI & & & 0.175 & \\
\hline$(-)$ & $52(52.0)$ & $14(47.7)$ & & $67(51.5)$ \\
\hline$(+)$ & $48(48.0)$ & $15(50.0)$ & & $63(48.5)$ \\
\hline unknown & $0(0.0)$ & $1(3.3)$ & & \\
\hline Resection margin & & & 0.868 & \\
\hline$(-)$ & 91 (91.0) & $27(90.0)$ & & $118(90.8)$ \\
\hline close or $(+)$ & $9(9.0)$ & $3(10.0)$ & & $12(9.2)$ \\
\hline Tumor grade & & & 0.297 & \\
\hline G1/G2 & $63(63.0)$ & $22(73.3)$ & & $85(65.4)$ \\
\hline G3 & $37(37.0)$ & $8(26.7)$ & & 45 (34.6) \\
\hline
\end{tabular}

Abbreviations: NR, nodal ratio; IDC, infiltrating ductal carcinoma; ER, estrogen receptor; PR, progesterone receptor; ECE, extracapsular extension; LVI, lymphovascular invasion; RM, resection margin

Table 3 Clinical status and patterns of failure

\begin{tabular}{|c|c|c|c|}
\hline & \multicolumn{2}{|c|}{ No. of patients (\%) } & \multirow[b]{2}{*}{$\begin{array}{c}\text { Total } \\
(n=130)\end{array}$} \\
\hline & $\begin{array}{l}N R \leq 0.15 \\
(n=100)\end{array}$ & $\begin{array}{c}N R>0.15 \\
(n=30)\end{array}$ & \\
\hline \multicolumn{4}{|l|}{ Follow-up (months) } \\
\hline median (range) & $61(10-89)$ & 48 (26-78) & $59(10-89)$ \\
\hline \multicolumn{4}{|l|}{ Clinical Status } \\
\hline NED & $96(96.0)$ & $26(86.6)$ & $122(93.9)$ \\
\hline alive with disease & $4(4.0)$ & $2(6.7)$ & $6(4.6)$ \\
\hline cause-specific death & $0(0.0)$ & $2(6.7)$ & $2(1.5)$ \\
\hline intercurrent death & $0(0.0)$ & $0(0.0)$ & $0(0.0)$ \\
\hline \multicolumn{4}{|l|}{ Patterns of failure } \\
\hline LRR only & $1(20.0)$ & $0(0.0)$ & $1(11.0)$ \\
\hline DM only & $3(60.0)$ & $1(25.0)$ & $4(44.5)$ \\
\hline $\mathrm{LRR}+\mathrm{DM}$ & $1(20.0)$ & $3(75.0)$ & $4(44.5)$ \\
\hline
\end{tabular}

Abbreviations: NR, nodal ratio; NED, no evidence of disease; LRR, locoregional recurrence; DM, distant metastasis 
Table 4 Univariate analysis for 5-year LRRFS, DMFS, and DFS

\begin{tabular}{|c|c|c|c|c|}
\hline Variable & No. of pts & $\begin{array}{r}5 \text {-year LRRFS } \\
(\%)\end{array}$ & $\begin{array}{r}5 \text {-year DMFS } \\
(\%)\end{array}$ & $\begin{array}{r}5 \text {-year DFS } \\
(\%)\end{array}$ \\
\hline \multicolumn{5}{|l|}{ Age } \\
\hline$\leq 40$ & 31 & 92.7 & 84.3 & 84.3 \\
\hline \multirow[t]{2}{*}{$>40$} & 99 & 96.8 & 96.2 & 93.7 \\
\hline & & $p=0.348$ & $p=0.078$ & $p=0.138$ \\
\hline \multicolumn{5}{|l|}{ T stage } \\
\hline $\mathrm{T} 1$ & 68 & 96.5 & 96.7 & 94.6 \\
\hline \multirow[t]{2}{*}{$T 2 / 3$} & 62 & 95.0 & 89.5 & 87.7 \\
\hline & & $p=0.578$ & $p=0.108$ & $p=0.229$ \\
\hline \multicolumn{5}{|l|}{ Nodal ratio } \\
\hline$\leq 0.15$ & 100 & 97.9 & 96.4 & 94.0 \\
\hline \multirow[t]{2}{*}{$>0.15$} & 30 & 88.7 & 81.3 & 81.3 \\
\hline & & $p=0.033$ & $p=0.029$ & $p=0.069$ \\
\hline \multicolumn{5}{|l|}{ ER } \\
\hline$(-)$ & 28 & 96.4 & 96.4 & 95.4 \\
\hline \multirow[t]{2}{*}{$(+)$} & 102 & 95.6 & 92.3 & 89.8 \\
\hline & & $p=0.895$ & $p=0.462$ & $p=0.374$ \\
\hline \multicolumn{5}{|l|}{$P R$} \\
\hline$(-)$ & 49 & 98.0 & 98.0 & 98.0 \\
\hline \multirow[t]{2}{*}{$(+)$} & 81 & 94.5 & 90.5 & 87.5 \\
\hline & & $p=0.403$ & $p=0.111$ & $p=0.087$ \\
\hline \multicolumn{5}{|l|}{ HER2 } \\
\hline$(-)$ or unknown & 107 & 94.4 & 92.0 & 89.7 \\
\hline \multirow[t]{2}{*}{$(+)$} & 23 & 100.0 & 100.0 & 100.0 \\
\hline & & $p=0.421$ & $p=0.180$ & $p=0.163$ \\
\hline \multicolumn{5}{|l|}{ Histologic grade } \\
\hline G1/G2 & 85 & 97.3 & 94.3 & 92.7 \\
\hline \multirow[t]{2}{*}{ G3 } & 45 & 93.1 & 91.7 & 89.2 \\
\hline & & $p=0.203$ & $p=0.354$ & $p=0.510$ \\
\hline \multicolumn{5}{|l|}{ ECE } \\
\hline$(-)$ or unknown & 97 & 95.5 & 91.3 & 90.2 \\
\hline \multirow[t]{2}{*}{$(+)$} & 33 & 96.8 & 100.0 & 95.2 \\
\hline & & $p=0.639$ & $p=0.117$ & $p=0.332$ \\
\hline \multicolumn{5}{|l|}{ LVI } \\
\hline (-) or unknown & 67 & 96.4 & 95.0 & 93.3 \\
\hline \multirow[t]{2}{*}{$(+)$} & 63 & 95.1 & 91.6 & 89.7 \\
\hline & & $p=0.639$ & $p=0.927$ & $p=0.768$ \\
\hline \multicolumn{5}{|l|}{ RM } \\
\hline$(-)$ & 118 & 96.2 & 94.9 & 92.7 \\
\hline \multirow[t]{2}{*}{ close or $(+)$} & 12 & 91.7 & 83.3 & 83.3 \\
\hline & & $p=0.431$ & $p=0.213$ & $p=0.268$ \\
\hline Surgery & & & & \\
\hline BCS & 67 & 98.5 & 95.5 & 95.5 \\
\hline MRM & 63 & 93.1 & 91.9 & 88.1 \\
\hline & & $p=0.160$ & $p=0.556$ & $p=0.345$ \\
\hline Regional RT & & & & \\
\hline no & 115 & 96.2 & 93.3 & 91.2 \\
\hline yes & 15 & 93.3 & 93.3 & 93.3 \\
\hline & & $p=0.503$ & $p=0.976$ & $p=0.935$ \\
\hline Chemotherapy & & & & \\
\hline no & 13 & 92.3 & 79.1 & 79.1 \\
\hline yes & 117 & 96.3 & 94.9 & 92.8 \\
\hline & & $p=0.401$ & $p=0.104$ & $p=0.173$ \\
\hline
\end{tabular}



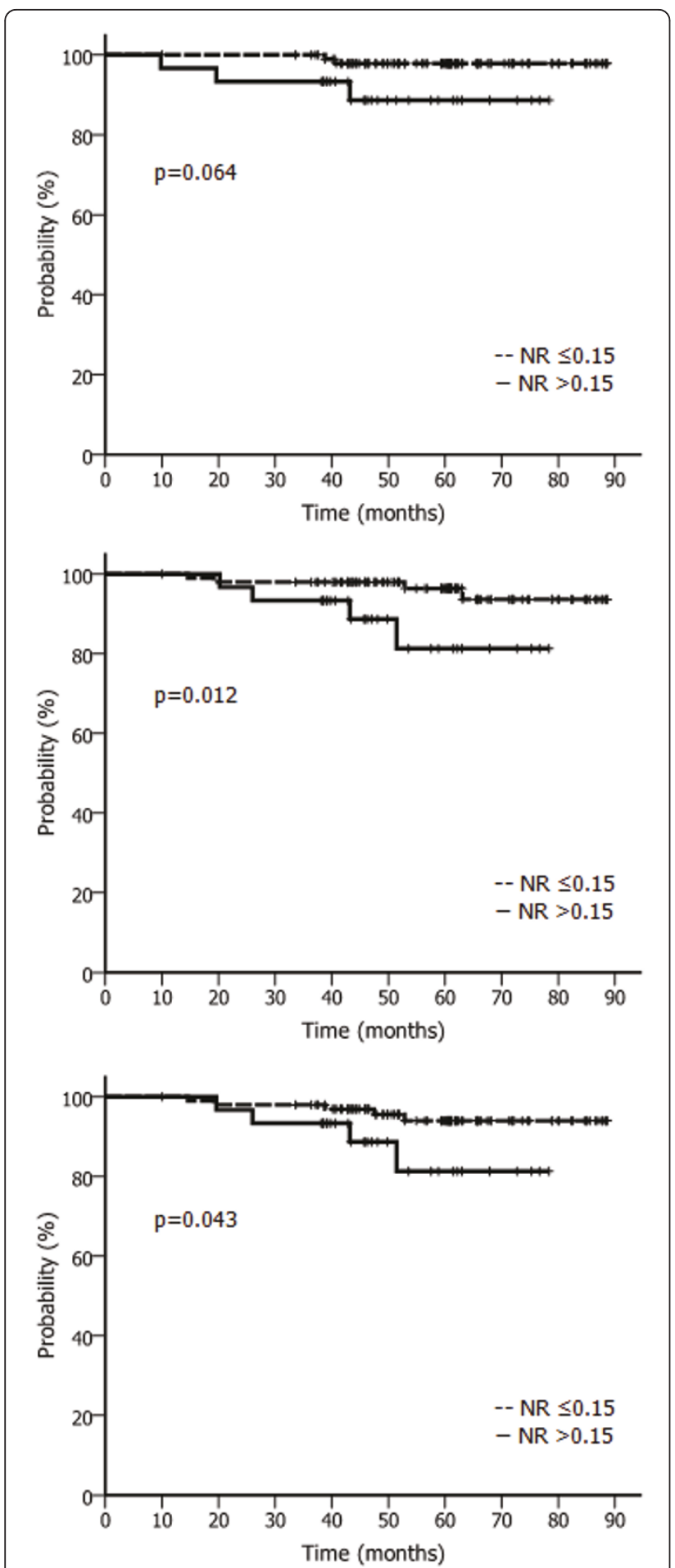

Figure 1 LRRFS, DMFS, and DFS according to NR. (a) LRRFS according to NR. (b) DMFS according to NR. (c) DFS according to NR. Abbreviations: LRRFS, locoregional recurrence-free survival; DMFS, distant metastasis-free survival; DFS, disease-free survival; NR, nodal ratio.
Table 5 Adjusted analysis for DFS

\begin{tabular}{|c|c|c|c|c|}
\hline & \multicolumn{3}{|c|}{ 5-year DFS (\%) } & \multirow[b]{2}{*}{$\mathrm{p}$ value } \\
\hline & No. of pts & $N R \leq 0.15$ & $N R>0.15$ & \\
\hline \multicolumn{5}{|l|}{ Age } \\
\hline$\leq 40$ & 31 & 90.4 & 53.3 & 0.027 \\
\hline$>40$ & 99 & 95.4 & 87.3 & 0.361 \\
\hline \multicolumn{5}{|l|}{ T stage } \\
\hline $\mathrm{T} 1$ & 68 & 95.4 & 90.9 & 0.586 \\
\hline $\mathrm{T} 2 / 3$ & 62 & 92.3 & 66.7 & 0.034 \\
\hline \multicolumn{5}{|l|}{ Histologic grade } \\
\hline $\mathrm{G} 1 / 2$ & 85 & 94.7 & 84.7 & 0.387 \\
\hline G3 & 45 & 92.6 & 75.0 & 0.034 \\
\hline \multicolumn{5}{|l|}{ LVI } \\
\hline$(-)$ or unknown & 67 & 94.0 & 87.5 & 0.849 \\
\hline$(+)$ & 63 & 93.8 & 75.8 & 0.037 \\
\hline \multicolumn{5}{|l|}{ RM } \\
\hline$(-)$ & 118 & 93.2 & 90.9 & 0.594 \\
\hline close or (+) & 12 & 100.0 & 33.3 & 0.007 \\
\hline \multicolumn{5}{|l|}{ Regional RT } \\
\hline no & 115 & 93.7 & 69.7 & 0.039 \\
\hline yes & 15 & 100.0 & 90.0 & 0.480 \\
\hline \multicolumn{5}{|l|}{ Chemotherapy } \\
\hline no & 13 & 100.0 & 80.0 & 0.014 \\
\hline yes & 117 & 93.4 & 90.7 & 0.556 \\
\hline
\end{tabular}

Abbreviations: DFS, disease-free survival; NR, nodal ratio; $E C E$, extracapsular extension; LVI, lymphovascular invasion; RM, resection margin

inadequate resection margins [15-19]. Recently, Truong et al. reported that $\mathrm{T} 1-\mathrm{T} 2$ breast cancer patients with one to three positive nodes, young age ( $<50$ years), histologic grade 3, or ER-negative disease had high 10-year locoregional recurrence risks (up to 20\%), even after breast-conserving surgery was followed by whole breast radiotherapy [15]. In the current study, those findings were not significant factors for locoregional recurrence or distant metastasis independently but showed selective significance in adjusted analysis combined with the NR.

Regardless of the extent of surgery, substantially high locoregional recurrence rates have been reported in patients with 1-3 positive nodes [15,20-24]. Locoregional recurrence also has been linked to distant metastasis and long-term breast cancer mortality [25-28]. In a meta-analysis of five National Surgical Adjuvant Breast and Bowel Project (NSABP) trials, patients who experienced locoregional recurrence had a considerably lower 5-year DMFS: 51.4\% after ipsilateral breast tumor recurrence, $31.5 \%$ after axillary recurrence, and $12.1 \%$ after supraclavicular metastasis, respectively [27]. Data from the Breast Cancer Trialists' Collaborative Group (BCTCG) showed the overall absolute reduction of 5year locoregional recurrence by $19 \%$, resulting in a $5 \%$ 

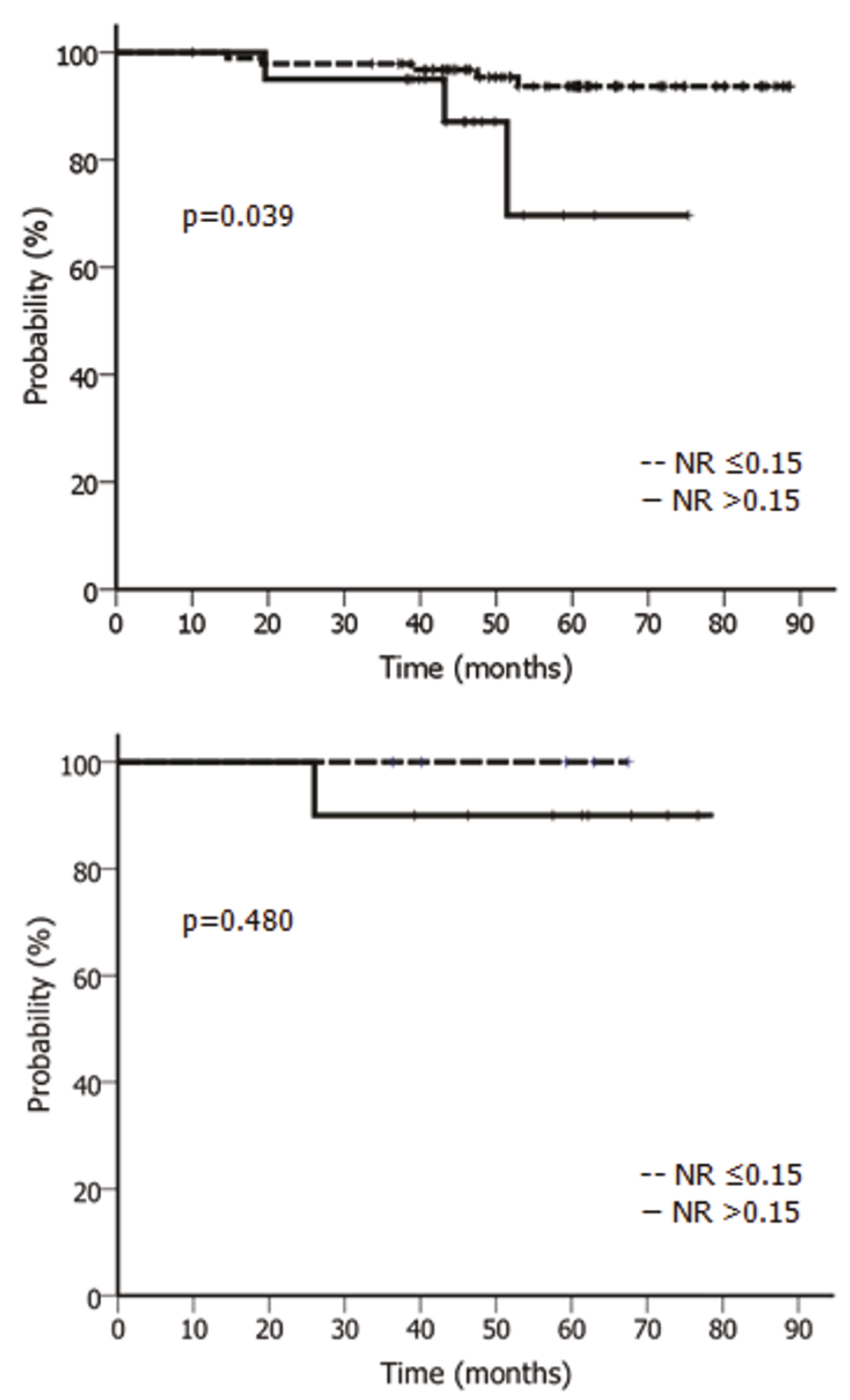

Figure 2 Adjusted analysis for DFS with or without regional RT. (a) DFS according to nodal ratio with regional RT. (b) DFS according to nodal ratio without regional RT. Abbreviations: DFS, disease-free survival; NR, nodal ratio.

overall absolute reduction of 15 -year breast cancer mortality risk in patients who underwent either breast-conserving surgery or mastectomy [28]. In the current study, the HNR group showed lower LRRFS, DMFS, and DFS. However, it is inconclusive whether decreased risk of distant metastasis resulted from decreased locoregional recurrence because only a small number of patients experienced locoregional recurrence.

The National Cancer Institute of Canada Clinical Trials Group (NCIC-CTG) has suggested that adding regional RT may improve survival compared with whole breast RT only when administered after breast-conserving surgery in patients who have T1-T2 breast cancer with N1 or moderate to high risk N0 [29]. The current study revealed that regional RT reduced the risk of distant metastasis in the HNR group only; however, this finding could also support the interpretation that regional RT is unnecessary for LNR patients who have undergone adequate axillary dissection and had no poor prognostic factors. For optimization of the locoregional modality, it is necessary to better define the selection criteria for adjuvant RT. The NR may be a useful indicator for deciding whether to use adjuvant regional RT to treat patients with $\mathrm{N} 1$ disease. 
Table 6 Multivariate analysis for LRRFS, DMFS, and DFS

\begin{tabular}{|c|c|c|c|}
\hline \multirow[b]{2}{*}{ Variables } & \multicolumn{3}{|l|}{ Hazard ratio $(95 \% \mathrm{Cl})$} \\
\hline & Locoregional recurrence & Distant metastasis & Any failure \\
\hline \multicolumn{4}{|l|}{$\overline{\text { Age }}$} \\
\hline \multirow[t]{2}{*}{$(>40$ vs. $\leq 40)$} & $0.62(0.07-5.27)$ & $0.07(0.01-0.80)$ & $0.22(0.04-1.36)$ \\
\hline & $p=0.664$ & $p=0.033$ & $p=0.104$ \\
\hline \multicolumn{4}{|l|}{ T stage } \\
\hline \multirow[t]{2}{*}{ (2-3 vs. 1) } & $1.02(0.11-9.02)$ & $6.09(0.83-44.72)$ & $2.58(0.52-12.82)$ \\
\hline & $p=0.989$ & $p=0.076$ & $p=0.247$ \\
\hline \multicolumn{4}{|l|}{ NR } \\
\hline \multirow[t]{2}{*}{$(>0.15$ vs. $\leq 0.15)$} & $7.38(0.89-61.25)$ & $11.22(1.70-74.04)$ & $5.06(1.05-24.37)$ \\
\hline & $p=0.064$ & $p=0.012$ & $p=0.043$ \\
\hline \multicolumn{4}{|l|}{ Histologic grade } \\
\hline \multirow[t]{2}{*}{ (3 vs. 1-2) } & $5.86(0.48-71.23)$ & $1.26(0.22-7.33)$ & $1.33(0.28-6.30)$ \\
\hline & $p=0.166$ & $p=0.795$ & $p=0.724$ \\
\hline \multicolumn{4}{|l|}{ LVI } \\
\hline \multirow[t]{2}{*}{ (positive vs. negative or unknown) } & $0.90(0.10-8.14)$ & $0.38(0.06-2.32)$ & $0.71(0.15-3.25)$ \\
\hline & $p=0.927$ & $p=0.294$ & $p=0.654$ \\
\hline \multicolumn{4}{|l|}{ RM } \\
\hline \multirow[t]{2}{*}{ (close or positive vs. negative) } & $1.36(0.08-22.32)$ & $1.87(0.22-16.26)$ & $1.62(0.23-11.30)$ \\
\hline & $p=0.829$ & $p=0.569$ & $p=0.624$ \\
\hline \multicolumn{4}{|l|}{ Surgery } \\
\hline \multirow[t]{2}{*}{ (MRM vs. BCS) } & $4.62(0.37-57.31)$ & $0.57(0.87-3.78)$ & $1.21(0.24-6.22)$ \\
\hline & $p=0.234$ & $p=0.563$ & $p=0.819$ \\
\hline \multicolumn{4}{|l|}{ Regional RT } \\
\hline \multirow[t]{2}{*}{ (yes vs. no) } & $1.70(0.12-24.18)$ & $0.64(0.06-6.95)$ & $0.59(0.06-5.92)$ \\
\hline & $p=0.695$ & $p=0.717$ & $p=0.653$ \\
\hline \multicolumn{4}{|l|}{ Chemotherapy } \\
\hline \multirow[t]{2}{*}{ (yes vs. no) } & $0.17(0.01-5.22)$ & $0.15(0.01-1.68)$ & $0.27(0.03-2.41)$ \\
\hline & $p=0.311$ & $p=0.125$ & $p=0.243$ \\
\hline
\end{tabular}

Abbreviations: Cl, confidence interval; LRRFS, locoregional recurrence-free survival; DMFS, distant metastasis-free survival; DFS, disease-free survival; LVI, lymphovascular invasion; RM, resection margin; BCS, breast-conserving surgery; MRM, modified radical mastectomy

Inadequate nodal sampling (less than 10), histology grade 3, estrogen receptor-negative breast carcinomas, or presence of LVI are all considered to be related to the risk of regional recurrence. Previous studies have shown that sampling fewer than 10 axillary nodes is associated with an increased risk of subsequent locoregional recurrence $[15,23,24,30]$. Tai et al. included in their study only patients with 10 or more excised nodes in order to avoid the possibility of an increased regional relapse rate resulting from understaging or undertreatment [6]. The adjuvant regional RT could compensate for the compromised regional control resulting from inadequate axillary dissection; however, this result does not directly apply to patients in the HNR group who have undergone adequate axillary dissection and remain at substantial risk for locoregional recurrence [31].

\section{Conclusions}

The results of this study associate a NR $>0.15$ with increased risk of disease recurrence, especially in young patients with unfavorable pathologic profiles.

\section{Abbreviations}

LN: Iymph node; BCS: breast-conserving surgery; MRM: modified radical mastectomy; SLNBx: sentinel lymph node biopsy; ALND: axillary lymph node dissection; CMF: cyclophosphamide/methotrexate/5-fluorouracil; FEC: 5-FU/ epirubicin/cyclophosphamide; FAC: 5-FU/adriamycin/cyclophosphamide; ACT: adriamycin/cyclophosphamide/paclitaxel; NR: nodal ratio; IDC: infiltrating ductal carcinoma; ER: estrogen receptor; PR: progesterone receptor; ECE: extracapsular extension; LVI: lymphovascular invasion; RM: resection margin NED: no evidence of disease; LRR: locoregional recurrence; DM: distant metastasis; LRRFS: locoregional recurrence-free survival; DMFS: distant metastasis-free survival; DFS: disease-free survival

\section{Acknowledgements}

This work was supported by Nuclear R\&D Program (BAERI\#2011-0006312) from National Research Foundation, Korean Ministry of Education, Science \& Technology and Cancer Control Program (\#0820010) from Korean Ministry of Health \& Welfare to Kim IA.

\section{Author details}

${ }^{1}$ Department of Radiation Oncology, Seoul National University, Bundang Hospital, 166 Gumiro Seongnamsi Kyeonggido, 463-707, Korea. ${ }^{2}$ Breast Care Center, Seoul National University, Bundang Hospital, Korea.

\section{Authors' contributions}

IAK designed this study and is responsible for the preparation of manuscript with TJH. TJH, EYK and WJ contributed to the management of clinical data. SWK, JHK, YJK, JSK, and IAK provided clinical expertise in clinical breast 
oncology. SYP contributed to the pathologic work. All authors read and approved the content of manuscript.

\section{Competing interests}

The authors declare that they have no competing interests.

Received: 8 June 2011 Accepted: 6 October 2011

Published: 6 October 2011

\section{References}

1. Vinh-Hung V, Burzykowski T, Cserni G, Voordeckers M, Van De Steene J, Storme G: Functional form of the effect of the numbers of axillary nodes on survival in early breast cancer. Int J Oncol 2003, 22:697-704.

2. American Joint Committee on Cancer: Chapter 32. Breast. AJCC cancer staging manual. 7 edition. New York: Springer; 2010, 347-369.

3. Recht A, Edge SB, Solin LJ, Robinson DS, Estabrook A, Fine RE, Fleming GF, Formenti S, Hudis C, Kirshner JJ, Krause DA, Kuske RR, Langer AS, Sledge GW Jr, Whelan TJ, Pfister DG, American Society of Clinical Oncology: Postmastectomy radiotherapy: Clinical practice guidelines of the American Society of Clinical Oncology. J Clin Oncol 2001, 19:1539-1569.

4. Woodward WA, Vinh-Hung V, Ueno NT, Cheng YC, Royce M, Tai P, Vlastos G, Wallace AM, Hortobagyi GN, Nieto Y: Prognostic value of nodal ratios in node-positive breast cancer. J Clin Oncol 2006, 24:2910-2916.

5. Truong PT, Berthelet E, Lee J, Kader HA, Olivotto IA: The prognostic significance of the percentage of positive/dissected axillary lymph nodes in breast cancer recurrence and survival in patients with one to three positive axillary lymph nodes. Cancer 2005, 103:2006-2014.

6. Tai $P$, Joseph $K$, Sadikov E, Mahmood S, Lien F, Yu E: Nodal ratios in nodepositive breast cancer-long-term study to clarify discrepancy of role of supraclavicular and axillary regional radiotherapy. Int J Radiat Oncol Biol Phys 2007, 68:662-666

7. Truong PT, Woodward WA, Thames HD, Ragaz J, Olivotto IA, Buchholz TA: The ratio of positive to excised nodes identifies high-risk subsets and reduces inter-institutional differences in locoregional recurrence risk estimates in breast cancer patients with 1-3 positive nodes: an analysis of prospective data from British Columbia and the M. D. Anderson Cancer Center. Int J Radiat Oncol Biol Phys 2007, 68:59-65.

8. Vinh-Hung V, Verkooijen HM, Fioretta G, Neyroud-Caspar I, Rapiti E, Vlastos G, Deglise C, Usel M, Lutz JM, Bouchardy C: Lymph node ratio as an alternative to $\mathrm{pN}$ staging in node-positive breast cancer. J Clin Oncol 2009, 27:1062-1068.

9. Hatoum HA, Jamali FR, El-Saghir NS, Musallam KM, Seoud M, Dimassi H, Abbas J, Khalife M, Boulos FI, Tawil AN, Geara FB, Salem Z, Shamseddine AA, Al-Feghali K, Shamseddine Al: Ratio between positive lymph nodes and total excised axillary lymph nodes as an independent prognostic factor for overall survival in patients with nonmetastatic lymph node-positive breast cancer. Ann Surg Oncol 2009, 16:3388-3395.

10. Danko ME, Bennett KM, Zhai J, Marks JR, Olson JA Jr: Improved staging in node-positive breast cancer patients using lymph node ratio: results in 1,788 patients with long-term follow-up. J Am Coll Surg 2010, 210:797-805

11. Schiffman SC, McMasters KM, Scoggins CR, Martin RC, Chagpar AB: Lymph node ratio: a proposed refinement of current axillary staging in breast cancer patients. J Am Coll Surg 2011, 213:45-52.

12. Chagpar AB, Camp RL, Rimm DL: Lymph Node Ratio Should Be Considered for Incorporation into Staging for Breast Cancer. Ann Surg Oncol

13. Ahn SH, Kim HJ, Lee JW, Gong GY, Noh DY, Yang JH, Jung SS, Park HY: Lymph node ratio and $\mathrm{pN}$ staging in patients with node-positive breast cancer: a report from the Korean breast cancer society. Breast Cancer Res Treat

14. Fortin A, Dagnault A, Blondeau L, Vu TT, Larochelle M: The impact of the number of excised axillary nodes and of the percentage of involved nodes on regional nodal failure in patients treated by breast-conserving surgery with or without regional irradiation. Int J Radiat Oncol Biol Phys 2006, 65:33-39.

15. Truong PT, Jones SO, Kader HA, Wai ES, Speers $\mathrm{CH}$, Alexander AS, Olivotto IA: Patients with T1 to T2 breast cancer with one to three positive nodes have higher local and regional recurrence risks compared with node-negative patients after breast-conserving surgery and whole-breast radiotherapy. Int J Radiat Oncol Biol Phys 2009, 73:357-364.

16. Wallgren A, Bonetti M, Gelber RD, Goldhirsch A, Castiglione-Gertsch M, Holmberg SB, Lindtner J, Thürlimann B, Fey M, Werner ID, Forbes JF, Price K, Coates AS, Collins J: Risk factors for locoregional recurrence among breast cancer patients: results from International Breast Cancer Study Group Trials I through VII. J Clin Oncol 2006, 24:2028-2037.

17. Katz A, Strom EA, Buchholz TA, Theriault R, Singletary SE, McNeese MD: The influence of pathologic tumor characteristics on locoregional recurrence rates following mastectomy. Int J Radiat Oncol Biol Phys 2001, 50:735-742

18. Veronesi U, Marubini E, Del Vecchio M, Manzari A, Andreola S, Greco M Luini A, Merson M, Saccozzi R, Rilke F: Local recurrences and distant metastases after conservative breast cancer treatments: partly independent events. Int J Radiat Oncol Biol Phys 2001, 50:735-742.

19. Yang PS, Chen CM, Liu MC, Jian JM, Horng CF, Liu MJ, Yu BL, Lee MY, Chi CW: Radiotherapy Can Decrease Locoregional Recurrence and Increase Survival in Mastectomy Patients with T1 to T2 Breast Cancer and One to Three Positive Nodes with Negative Estrogen Receptor and Positive Lymphovascular Invasion Status. Int J Radiat Oncol Biol Phys 2010, 77:516-522.

20. Recht A, Gray R, Davidson NE: Locoregional failure 10 years after mastectomy and adjuvant chemotherapy with or without tamoxifen without irradiation: Experience of the Eastern Cooperative Oncology Group. J Clin Oncol 1999, 17:1689-1700.

21. Katz A, Strom EA, Buchholz TA, Thames HD, Smith CD, Jhingran A, Hortobagyi G, Buzdar AU, Theriault R, Singletary SE, McNeese MD: Locoregional recurrence patterns after mastectomy and doxorubicinbased chemotherapy: Implications for postoperative irradiation. J Clin Oncol 2000, 18:2817-2827.

22. Woodward WA, Strom EA, Tucker SL, Katz A, McNeese MD, Perkins GH, Buzdar AU, Hortobagyi GN, Hunt KK, Sahin A, Meric F, Sneige N, Buchholz TA: Locoregional recurrence after doxorubicinbased chemotherapy and postmastectomy: Implications for breast cancer patients with early-stage disease and predictors for recurrence after postmastectomy radiation. Int J Radiat Oncol Biol Phys 2003, 57:336-344.

23. Truong PT, Olivotto IA, Kader HA, Panades $M$, Speers $C H$, Berthelet $E$ : Selecting breast cancer patients with T1-T2 tumors and one to three positive axillary nodes at high postmastectomy locoregional recurrence risk for adjuvant radiotherapy. Int J Radiat Oncol Biol Phys 2005, 61:1337-1347.

24. Lukens JN, Vapiwala N, Hwang WT, Solin LJ: Regional nodal recurrence after breast conservation treatment with radiotherapy for women with early-stage breast carcinoma. Int J Radiat Oncol Biol Phys 2009, 73:1475-1481.

25. Buchholz TA, Woodward WA, Duan Z, Fang S, Oh JL, Tereffe W, Strom EA, Perkins GH, Yu TK, Hunt KK, Meric-Bernstam F, Hortobagyi GN, Giordano SH: Radiation use and long-term survival in breast cancer patients with T1, $\mathrm{T} 2$ primary tumors and one to three positive axillary lymph nodes. Int Radiat Oncol Biol Phys 2008, 71:1022-1027.

26. Whelan TJ, Julian J, Wright J, Jadad AR, Levine ML: Does locoregional radiation therapy improve survival in breast cancer? A meta-analysis. $J$ Clin Oncol 2000, 18:1220-1229.

27. Wapnir IL, Anderson SJ, Mamounas EP, Geyer CE Jr, Jeong JH, Tan-Chiu E, Fisher B, Wolmark N: Prognosis after ipsilateral breast tumor recurrence and locoregional recurrences in five National Surgical Adjuvant Breast and Bowel Project node-positive adjuvant breast cancer trials. J Clin Oncol 2006, 24:2028-2037.

28. Clarke M, Collins R, Darby S, Davies C, Elphinstone P, Evans E, Godwin J, Gray R, Hicks C, James S, MacKinnon E, McGale P, McHugh T, Peto R, Taylor C, Wang Y, Early Breast Cancer Trialists' Collaborative Group (EBCTCG): Effects of radiotherapy and of differences in the extent of surgery for early breast cancer on local recurrence and 15-year survival: an overview of the randomised trials. Lancet 2005, 366:2087-2106.

29. Olivotto IA, Chua B, Elliott EA, Parda DS, Pierce LJ, Shepherd L, Vallow LA, White JR, Whelan TJ: A clinical trial of breast radiation therapy versus breast plus regional radiation therapy in early-stage breast cancer: the MA20 trial. Clin Breast Cancer 2003, 4:361-363.

30. Joslyn SA, Konety BR: Effect of axillary lymphadenectomy on breast carcinoma survival. Breast Cancer Res Treatment 2005, 91:11-18. 
31. Fowble B: Postmastectomy radiation in patients with one to three positive axillary nodes receiving adjuvant chemotherapy: An unresolved issue. Semin Radiat Oncol 1999, 9:230-240.

doi:10.1186/1748-717X-6-131

Cite this article as: Han et al:: The prognostic value of the nodal ratio in N1 breast cancer. Radiation Oncology 2011 6:131.

Submit your next manuscript to BioMed Central and take full advantage of:

- Convenient online submission

- Thorough peer review

- No space constraints or color figure charges

- Immediate publication on acceptance

- Inclusion in PubMed, CAS, Scopus and Google Scholar

- Research which is freely available for redistribution

Submit your manuscript at www.biomedcentral.com/submit 